\title{
Finite-Time Stability Control of Permanent Magnet Synchronous Motor Chaotic System Based on Lyapunov Function Method
}

\author{
Shuilong Zou ${ }^{1, a^{\star}}$ and Wenjie Zhang ${ }^{2, b}$ \\ ${ }^{1}$ Nanchang Institute of Science and Technology, Nanchang, 330108, China \\ a752782167@qq.com, ${ }^{b} 568740355 @ q q . c o m$
}

Keywords: PMSM; Chaos control; Control lyapunov function; Finite-time stability theory

\begin{abstract}
Permanent magnet synchronous motors ( PMSM) will demonstrate the complex dynamic behaviors under certain parameters and operation conditions. The performance of PMSM will degrade because of dynamic behaviors, a method of controlling chaos by designing a robust finite-time stability controller is developed to guide chaotic motion towards regular motion. The controller combines with Controlling Lyapunov Function method and finite-time stability theory. The controller not only has strong robustness and good responsiveness but also is simple and easy to be realized with few parameters processing. The simulation results show that the method of controlling permanent magnet synchronous motor chaotic system is effective.
\end{abstract}

\section{Introduction}

With the development of power electronics technology and control technology, permanent magnet synchronous motor (PMSM) will be widely used in industry .Chaos in the permanent magnet synchronous motor and its control is a area of active research in the field of numerical control machine.The permanent magnet synchronous motor is a kind of typical multivariable and strong coupling nonlinear system and presents chaotic behavior in some parameters and working conditions, main performance for the torque and speed of intermittent oscillations, system of irregular electromagnetic noise, etc. These irregular movements affect the stable operation of the system, how to eliminate the chaotic behavior of permanent magnet synchronous motor system has become the attention control subject.

\section{Mathematical Model of PMSM and Chaotic Indicator}

The dynamics of PMSM can be modeled, based on the d-q axis, where $i_{d}, i_{q}$ and $\omega$ are the state variables, which represent currents and motor angular frequency, respectively, $u_{d}$ and $u_{q}$ the direct- and quadrature-axis stator voltage components, respectively, $J$ the polar moment of inertia, $T_{L}$ the external load torque, $\beta$ the viscous damping coefficient, $R_{1}$ the stator winding resistance, $L_{d}$ and $L_{q}$ the directand quadrature-axis stator inductors, respectively, $\psi_{r}$ the permanent magnet flux, and $n_{p}$ the number of pole-pairs.

$$
\left\{\begin{array}{l}
\frac{d i_{d}}{d t}=\left(u_{d}-R_{1} i_{d}+\omega L_{q} i_{q}\right) / L_{q} \\
\frac{d i_{q}}{d t}=\left(u_{q}-R_{1} i_{q}-\omega \psi_{r}\right) / L_{q} \\
\frac{d \omega}{d t}=\left[n_{p} \psi_{r} i_{q}+n_{p}\left(L_{d}-L_{q}\right) i_{d} i_{q}-T_{L}-\beta \omega\right] / J
\end{array}\right.
$$

By applying an affine transformation $x=\lambda \tilde{x}$ and a time-scaling transformation $t=\tau \tilde{t}$ 


$$
\left\{\begin{array}{l}
\frac{d \tilde{i_{d}}}{d t}=-\tilde{i_{d}}+\tilde{\omega} \tilde{i_{q}}+\tilde{u_{d}} \\
\frac{d \tilde{i}_{q}}{d t}=-\tilde{i_{q}}+\tilde{\omega} \tilde{i_{d}}+\tilde{\gamma \omega}+\tilde{u_{d}} \\
\frac{\tilde{\omega}}{d t}=\sigma\left(\tilde{i_{q}}-\tilde{\omega}\right)+\varepsilon \tilde{\varepsilon} \tilde{i_{d}} \tilde{i}_{q}-T_{L}
\end{array}\right.
$$

we only study the dynamic characteristics of the PMSM, namely, $L_{d}=L_{q}=L$ in the model. Thus, Eq 1 becomes

$$
\left\{\begin{array}{c}
\dot{x}_{1}=-x_{1}+x_{3} x_{2}+\tilde{u}_{d} \\
\dot{x}_{2}=-x_{2}-x_{3} x_{1}+\gamma x_{3}+\tilde{u}_{q} \\
\dot{x}_{3}=\sigma\left(x_{2}-x_{3}\right)-\tilde{T}_{L}
\end{array}\right.
$$

$\tilde{u}_{d}=\tilde{u}_{q}=\tilde{T}_{L}=0$, this case can be thought of as that, after an operating period of the system, the external inputs are set to zero. Thus, Eq 3 becomes

$$
\left\{\begin{array}{c}
\dot{x}_{1}=-x_{1}+x_{3} x_{2} \\
\dot{x}_{2}=-x_{2}-x_{3} x_{1}+\gamma x_{3} \\
\dot{x}_{3}=\sigma\left(x_{2}-x_{3}\right)
\end{array}\right.
$$

When Runge-Kutta-Fehlberg integrator of variable time steps, is used to integrate the Eq 1, we obtain the chaotic attractor in Fig1, where initial condition $\left(\tilde{i}_{d}, \tilde{i}_{q}, \tilde{\omega}\right)=(20.0 .01,-5)$ and $(\sigma, \gamma)=(5.46,20)$.

The Eq 5 as particular solutions of the system satisfy the following algebraic equations:

$$
\left\{\begin{array}{c}
-\tilde{i}_{d}+\tilde{\omega} \tilde{i}_{q}=0 \\
-\tilde{i}_{q}-\tilde{\omega} \tilde{i}_{d}+\mu \tilde{\omega}=0 \\
\sigma\left(\tilde{i}_{q}-\tilde{\omega}\right)=0
\end{array}\right.
$$




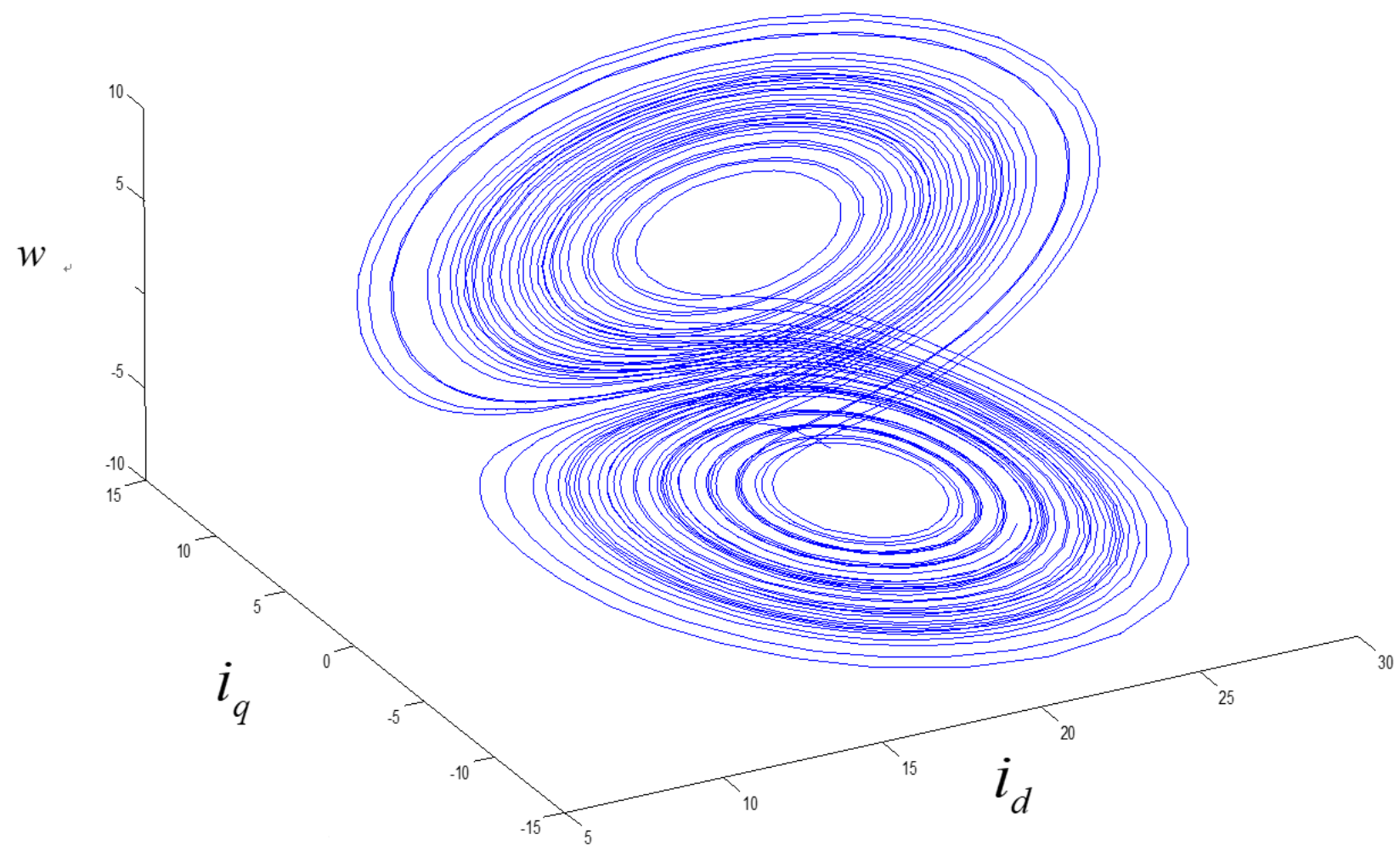

Fig 1. Strange attractor in PMSM

Obviously, $\tilde{i}_{d}^{e q}=\mu-1, \tilde{i}_{q}^{e q}= \pm \sqrt{\mu-1}, w^{e q}= \pm \sqrt{\mu-1}$ are the equilibrium points. At the point $\tilde{i}_{q}^{e q}$, the Jacobian matrix of the linearized equations of the Eq 6 is written as

$$
J_{1}=\left(\begin{array}{ccc}
-1 & \sqrt{\mu-1} & \sqrt{\mu-1} \\
-\sqrt{\mu-1} & -1 & 1 \\
0 & \sigma & -\sigma
\end{array}\right)
$$

On the one hand, all the eigenvalues of the characteristic equations have negative real parts which display the system stable when $\mu<14.93$, On the other hand there are always two eigenvalues with positive real parts as $\mu>14.93$. Namely, the stability of the existing equilibrium changes from being stable to unstable as the parameter $\mu$ is varied to pass the critical value 14.93 . Thus, we can claim that a Hopf bifurcation occurs at $\mu=14.93$. As a point to emphasize, one pair of purely imaginary eigenvalues and all other eigenvalues having negative real parts are sufficient

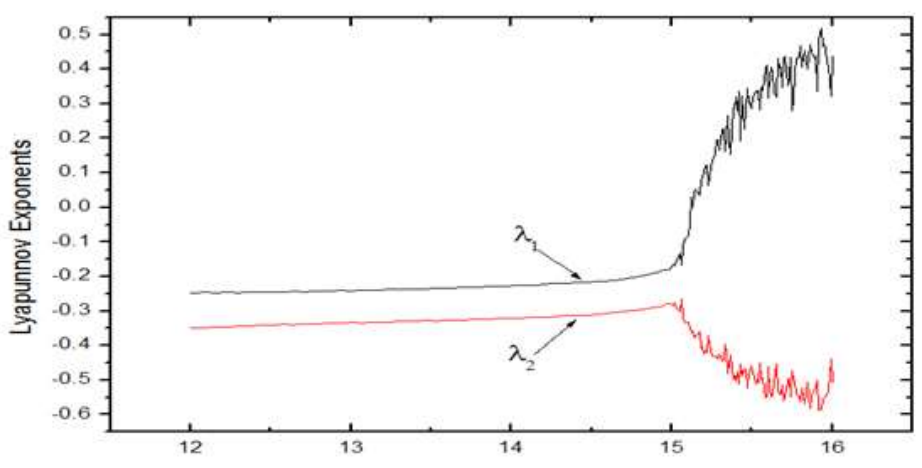

Fig 2. The Lyapunov spectra 
For the occurrence of a Hopf bifurcation at a certain critical parameter value. When the control forces put into controlling at any time, it is stable at some equilibrium points, The Lyapunov spectra is shown in fig. 2 .

\section{Numerical Simulation and Analysis}

In order to confirm the effectiveness of the adopted method and the feasibility of the controller design,We make use of the MATLAB simulation to PMSM chaos system. We adopt four order Runge Kutta algorithm in the simulation process.

For without loss of generality, this paper sets the system initial $X(0)=\left[x_{1}(0), x_{2}(0), x_{3}(0)\right]=[0.1,0.1,0.1]$, parameters of the controller selected as $\beta=3, \eta=1 / 3$ and $\beta=4, \eta=1 / 2$, set references PMSM chaotic system parameters. Fig 3, Fig 4 show the response curve of PMSM chaotic system controller role in the design of this article, at $t=20 \mathrm{~s}$ when added to the controller role.

As it can be seen from Fig 3 and Fig 4, compared with the program of the literature, this article can be reached quickly without overshoot equilibrium point of the system. However, the program has less control parameters than the literature, and keeps convenient and flexible. Fig 3, Fig 4 also show that choosing different controller parameters can change the system overshoot and response time. The simulation results show that the controller not only has strong robustness and short response time, but also relatively few parameters of the controller .

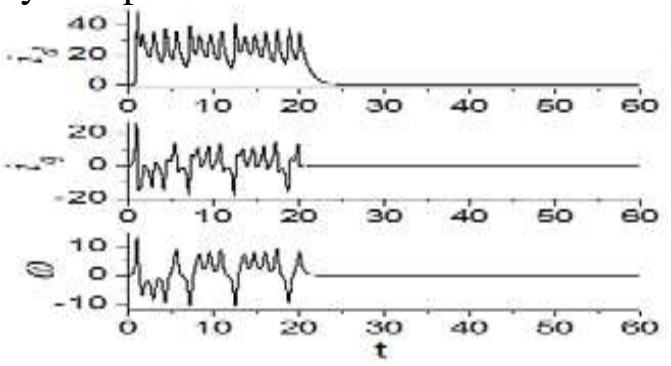

Figure 3 Control parameters $\beta=3, \eta=\frac{1}{2}$ the system response curve.

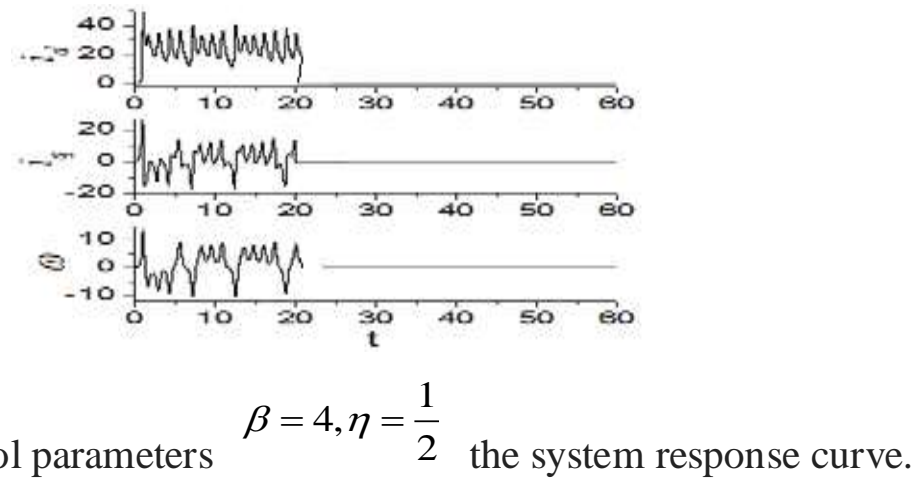

\section{Conclusion}

On the basis of combined controlling Lyapunov function method and finite time control theory, this paper presents a robust finite time stability controller, which can effectively control the movement of the permanent magnet synchronous motor chaos. On the basis of ensuring a strong robustness and short response time, compared with the previous controller, the parameters of the controller requires less and the algorithm is simple and easy to implement. Simulation results show the effectiveness of the controlled method. 


\section{Acknowledgements}

Fund projects: The research topic of teaching reform in Jiangxi higher schools(JXJG-16-27-11), The research topic of teaching reform in Nanchang Institute of Technology(NGJG-2015-34).

This work was supported in part by the National Science Foundation of China (51765042, 61463031, 61662044, 61773051), Jiangxi Provincial Department of Science and Technology (20142BBE50037, 20151BBE50050), and Jiangxi Provincial Department of Education (GJJ151236, JXYJG-2017-020, GJJ151234, GJJ161225).

\section{Reference}

[1]D. Liu, H. P. Ren, X. Y. Liu, "Chaos control in permanent magnet synchronous motors", IEEE Circuits and Systems, ISCAS'04, Vol. 4, pp. 732 735, May.2014

[2]Hassan Salarieh, Aria Alasty, "Adaptive chaos synchronization in Chua's systems with noisy parameters", Mathematics and Computers in Simulation,Vol.79, No.3, pp. 233 241, 2008.

[3]M.T. Yassen, "Controlling chaos and synchronization for new chaotic system using linear feedback control", Chaos, Solitons and Fractals Vol.26, No.3, pp. 913 920, 2015

[4]Tang Chuan-Sheng, Dai Yue-Hong.Finite-time stability control of permanent magnet synchronous motor with parameters uncertain[J]. Acta Physica Sinica,2013.

[5]WANG Xing-yuan,DUAN Chao-feng. Chaos control ling of uncertain Lorenz system using linear feedback[J]. Journal of Dalian University of Technology, 2015,45(6):892-896.

[6]WANG Lei,LI Ying-hui,ZHU Xi-hua,ZHANG Jing. Chaos synchronization of permanent magnet synchronous motor with disturbance using fuzzy adaptive logic[J]. Power System Protection and Control, 2011,39(11):33-43.

[7]Wei Du-Qu, Luo Xiao-Shu,Fang Jin-Qing,Wang Bing-Hong. Controlling chaos in permanent magnet synchronous motor based on the differential geometry method[J]. Acta Physica Sinica, 2006, 55(1):54-59.

[8]WU Zhong-qiang, Tan Fu-xiao. Passivity Control of Permanent-magnet Synchronous Motors Chaotic System[J]. Proceedings of the CSEE, 2006,26(18):159-163.

[9] Yongfeng Zheng. Research on project driven curriculum system of Mechatronics major in Higher Vocational Education[D]. Zhejiang Normal University, 2014.05.

[10] Caibing $\mathrm{Wu}$. Tungsten resource development project driving practice teaching course[M]. Metallurgical industry press, 2016.08. 